Pacific Journal of Mathematics

ON JUNG'S CONSTANT AND RELATED CONSTANTS IN 


\title{
ON JUNG'S CONSTANT AND RELATED CONSTANTS IN NORMED LINEAR SPACES
}

\author{
DAN AMIR
}

\begin{abstract}
In this paper several results on certain constants related to the notion of Chebyshev radius are obtained. It is shown in the first part that the Jung constant of a finite-codimensional subspace of a space $C(T)$ is 2, where $T$ is a compact Hausdorff space which is not extremally disconnected. Several consequences are stated, e.g. the fact that every linear projection from a space $C(T), T$ a perfect compact Hausdorff space, onto a finite-codimensional proper subspace has norm at least 2 .

The second discusses mainly the "self-Jung constant" which measures "uniform normal structure." It is shown that this constant, unlike Jung's constant, is essentially determined by the finite subsets of the space.
\end{abstract}

1. Jung constant in $C(T)$ spaces. For a bounded subset $A$ of a normed linear space $E$ and a subset $Y$ of $E$ we denote by $\operatorname{diam} A$ the diameter of $A$ ( $\left.\sup _{x, y \in A}\|x-y\|\right)$, by $r_{Y}(A)$ the relative Chebyshev radius of $A$ with respect to $Y\left(\inf _{y \in Y} \sup _{x \in A}\|x-y\|\right)$, and by $Z_{Y}(A)$ the relative Chebyshev center set of $A$ in $Y\left(\left\{y \in Y\right.\right.$; $\left.\left.\sup _{x \in A}\|x-y\|=r_{Y}(A)\right\}\right)$. The Jung constant of $E$ is $J(E)=\sup \left\{2 r_{E}(A) ; A \subset E\right.$, $\left.\operatorname{diam} A=1\right\}$. It is easily seen that $1 \leqq J(E) \leqq 2$. For $n$-dimensional spaces $E_{n}$, it was shown by Jung [12] that $J\left(l_{2}^{n}\right)=(2 n /(n+1))^{1 / 2}$ and $J\left(E_{n}\right)=1$ if and only if $E_{n}=l_{\infty}^{n}$. Bohnenblust [2] showed that $J\left(E_{n}\right) \leqq 2 n /(n+1)$, and Leichtweiss [14] characterized the extremal case (in the 2-dimensional case it is the hexagonal plane). In the infinite-dimensional case, it was shown that $J\left(l_{2}\right)=\sqrt{2}$ (Routledge [20]), and that $J(E)=1$ if and only if $E=C(T)$ for a Stonian $T$, i.e. if $E \in \mathscr{P}_{1}$ (Davis [5]) (cf. also [10], pages 91-92 in [11] and $\S 6$ in [4]).

Studying intersections of balls with subspaces, Franchetti [6] deduced that for every finite-codimensional subspace $E$ of $C[a, b]$ we have $J(E) \geqq$ $3 / 2$. A stronger and more general result is true.

1.1. Proposition. If the compact Hausdorff space $T$ is not extremally disconnected, then for every finite-codimensional subspace $E$ of $C(T)$ we have $J(E)=2$. 
We need the following

1.2. LemMA. Let $E$ be a finite-codimensional subspace of $C(T), T$ compact Hausdorff. Then for every $\varepsilon>0$ and every infinite open $V \subset T$ there is $f \in E$ with $\|f\|=1, f(T \backslash V)=0$ and $f \geqq-\varepsilon$.

Proof of the lemma. In the case where $V$ contains no isolated points, the proof is quite short: Since $V$ is infinite, $\{f \in E ; f(T \backslash V)=0\}$ is infinite dimensional and there are $f_{1} \in E, t_{1} \in V$ with $\left\|f_{1}\right\|=1=f_{1}\left(t_{1}\right)$, $f_{1}(T \backslash V)=0$. For $V_{1}=\left\{t \in V ; f_{1}(t)>1-\varepsilon\right\}$, which is infinite too, find in the same way $f_{2} \in E, t_{2} \in V_{1}$ with $\left\|f_{2}\right\|=1=f_{2}\left(t_{2}\right), f_{2}\left(T \backslash V_{1}\right)=$ 0 , etc. $g=\sum_{j=1}^{n} f_{j}$ satisfies $\|g\| \geqq g\left(t_{n}\right)>n(1-\varepsilon)$, while, since $f_{j}(t)<0$ happens only when $f_{j-1}(t)>1-\varepsilon$ and $f_{j+1}(t)=0, g(t)>-\varepsilon$. Normalize to get $f$.

For the general case we apply

1.3. Sublemma. Given an infinite matrix $\left(x^{j}(k)\right)_{j=1, \ldots, n ; k=1,2, \ldots}$ such that $x^{j}(k) \rightarrow 0$ as $k \rightarrow \infty$ for $j=1, \ldots, n$ and $\varepsilon>0$, there are $k$ and $\left(\varepsilon_{i}\right)_{i=1}^{\infty}$ such that $\left|\varepsilon_{i}\right| \leqq \varepsilon$ for all $i$ and $x^{j}(k)=\sum_{i \neq k} \varepsilon_{i} x^{j}(i)$ for $j=1, \ldots, n$.

Proof of the sublemma. We may assume that the rows $x^{1}, \ldots, x^{n}$ are linerly independent. Therefore there are also $n$ independent columns, which we may assume to be the first $n$ ones. Let $\left(\gamma_{r, s}\right)_{r, s=1}^{n}$ be the inverse of the matrix $\left(x^{j}(k)\right)_{j, k=1}^{n}$ and $c=\sum_{r, s}\left|\gamma_{r, s}\right|$. There is $k$ such that $\left|x^{j}(k)\right|<$ $\varepsilon / c$ for $j=1, \ldots, n$. Represent the $k$ th column as a linear combination of the first $n$ ones.

Proof of the lemma in the general case. Take a sequence $\left(f_{k}\right)_{k=1}$ of disjointly supported nonnegative norm-one functions sitting in $V$. Apply the sublemma to $x^{J}(k)=\mu_{j}\left(f_{k}\right)$, where $\mu_{1}, \ldots, \mu_{n} \in C(T)^{*}$ are such that $E=\left\{\mu_{1}, \ldots, \mu_{n}\right\}_{\perp}$. Take $f=f_{k}-\sum_{i \neq k} \varepsilon_{i} f_{i}$.

Proof of the proposition. Choose disjoint open subsets $V_{1}, V_{2}$ and $w \in \bar{V}_{1} \cap \bar{V}_{2}$ (such $w, V_{1}, V_{2}$ exist since $T$ is not extremally disconnected). Fix $\varepsilon>0$. Let $A \subset E$ consist of all $f_{1}-f_{2}$, when $f_{i}$ run over all the functions $f$ satisfying the conclusions of the lemma with respect to $V_{i}$. Then $f^{*}=\sup _{f \in A} f$ is 1 on $V_{1}$ and $\leqq \varepsilon$ on $V_{2}$, while $f_{*}=\inf _{f \in A} f$ is -1 on $V_{2}$ and $\geqq-\varepsilon$ on $V_{1}$. Thus the diameter of $A$ is $\leqq 1+\varepsilon$. The radius of $A$, however, is $\geqq 1$ since $\max _{t_{1}, t_{2} \in V}\left|f^{*}\left(t_{1}\right)-f_{*}\left(t_{2}\right)\right|=2$ in every neighborhood $V$ of $w$. 
REMARK. Proposition 1.1 verifies also a conjecture of Franchetti ([7]): If $J(C(T))<2$ then $T$ is extremally disconnected (and then $J(C(T))=1$ by Davis' result). This last result has been proved independently by $C$. Franchetti [8].

Lemma 1.2 can be applied also to improve Proposition 2 in [6], giving an alternative proof of our Proposition 1.1 in the perfect case.

1.4. Proposition. Let $F$ be a finite-codimensional subspace of $C(T), T$ perfect compact Hausdorff space. Then for every $x \in C(T)$ and every $s>d \equiv d(x, F)$ we have

$$
Z_{F}(B(x, s) \cap F)=P_{F} x \text { and } r_{F}(B(x, s) \cap F)=s+d,
$$

where $B(x, s)$ is the closed s-ball centered at $x(\{y ;\|y-x\| \leqq s\})$ and $P_{F} x$ । is the best approximation to $x$ in $F$.

Proof. Given any $y_{0} \in F$ with $\left\|x-y_{0}\right\|>d$, we want to show that there is a $y \in F$ with $\|x-y\| \leqq s$ and $\left\|y-y_{0}\right\|>s+d$. This will establish both claims, since if $\left\|x-y_{1}\right\|<d+\varepsilon$ then clearly $\left\|y-y_{1}\right\| \leqq \| y-$ $x\|+\| x-y_{1} \|<s+d+\varepsilon$ for every such $y$.

Without loss of generality we may assume $y_{0}=0,\|x\|=x\left(t_{0}\right)$ for some $t_{0} \in T$. If $\|x\|<s$, let

$$
0<\varepsilon<\min \left(\frac{s-\|x\|}{s+d+1}, \frac{\|x\|-d}{2}, 1\right),
$$

$V=\left\{t ;\left|x(t)-x\left(t_{0}\right)\right|<\varepsilon\right\}$. Apply Lemma 1.2 to get $z \in F$ with $\|z\|=1$ and $z \geqq-\varepsilon$ which vanishes off $V$. Let $y=(s+d+\varepsilon) z$. Clearly $\|y\|>s$ $+d$. If $t \notin V$, then $|(x-y)(t)|=|x(t)| \leqq\|x\|<s$. If $t \in V$ then

$$
-s<\|x\|-\varepsilon-(s+d+\varepsilon) \leqq(x-y)(t) \leqq\|x\|+\varepsilon(s+d+\varepsilon)<s .
$$

If $\|x\| \geqq s$, let $y_{1} \in F$ satisfy $d<\left\|x-y_{1}\right\|<s$. Let

$$
0<\varepsilon<\min \left(\frac{s-\left\|x-y_{1}\right\|}{s+d}, \frac{\left\|s-y_{1}\right\|-d}{2}, y_{1}\left(t_{0}\right)\right) \text {, }
$$

$V=\left\{t:\left|x(t)-x\left(t_{0}\right)\right|+\left|y_{1}(t)-y_{1}\left(t_{0}\right)\right|<\varepsilon\right\}$. Apply Lemma 1.2 to get $z \in F$ with $\|z\|=1=z\left(t_{1}\right), z \geqq-\varepsilon$ which vanishes off $V$. Let $y=y_{1}+$ $(s+d) z$.

$$
\|y\| \geqq y_{1}\left(t_{1}\right)+s+d>y_{1}\left(t_{0}\right)-\varepsilon+s+d>s+d .
$$

If $t \notin V$, then $|(x-y)(t)|=\left|\left(x-y_{1}\right)(t)\right|<s$. If $t \in V$, then

$$
\begin{aligned}
-s & <\left(x-y_{1}\right)\left(t_{0}\right)-\varepsilon-s-d \\
& \leqq(x-y)(t) \leqq\left(x-y_{1}\right)(t)+(s+d) \varepsilon<s .
\end{aligned}
$$


1.5. Corollary. If $F$ is a subspace of $C(T), T$ any compact Hausdorff space with no isolated points, and $1 \leqq \operatorname{codim} F<\infty$, then $J(F)=2$.

Thus, for perfect $T$, the restriction in Proposition 1.1 that $T$ be non-Stonian is necessary (for $J(F)=2$ ) only in the case $F=C(T)$. Further concessions are impossible - since if $t_{0} \in T$ is isolated in the Stonian space $T$, then $F=\left\{x \in C(T) ; x\left(t_{0}\right)=0\right\}$ is isometric to $C\left(T^{\prime}\right)$, where $T^{\prime}=T \backslash\left\{t_{0}\right\}$ is Stonian too, hence $J(F)=1$.

Applying Franchetti's observation on the relation between projection constants of hyperplanes and radii of hypercircles [8], we get:

1.6. COROllaRY. If $F$ is a finite-codimensional proper subspace of $C(T), T$ perfect compact Hausdorff space, then every linear projection of $C(T)$ onto $F$ has norm $\geqq 2$.

Proof. Let $F=\left\{\mu_{1}, \ldots, \mu_{n}\right\}_{\perp}, \quad \mu_{i} \in C(T)^{*}, \quad\left\|\mu_{i}\right\|=1, \quad E=$ $\left\{\mu_{1}, \ldots, \mu_{n-1}\right\}_{\perp}$ such that $F$ is a maximal subspace of $E$. A linear projection of $E$ onto $F$ has the form $P x=x-\mu_{n}(x) z$, where $z \in E$ and $\mu_{n}(z)=1$. But

$$
\begin{aligned}
\|P\| & \geqq \sup _{0 \leqq \alpha<1} \sup _{\|x\| \leqq 1}\|P x\|=\sup _{0 \leqq \alpha<1} \sup _{\substack{y \in F \\
\|y+\alpha z\| \leqq 1}}\|y\| \\
& \geqq \sup _{0 \leqq \alpha<1} r_{F}(B(-\alpha z, 1) \cap F) .
\end{aligned}
$$

By Proposition 1.4, since $d(-\alpha z, F)=\alpha, r_{F}(B(-\alpha z, 1) \cap F)=1+\alpha$, so that $P=\sup _{0 \leqq \alpha<1}(1+\alpha)=2$.

Thus, every projection of $E$ onto $F$, and therefore also every projection of $C(T)$ onto $f$, has norm $\geqq 2$.

2. Jung constants and normal structure coefficients. By a classical result of Garkavi and Klee (cf., e.g. [13]) $r_{A}(A)=r(A)$ for all convex closed and bounded $A \subset E$ is equivalent to $E$ having dimension $\leqq 2$ or being an inner product space. Therefore, besides the Jung constant $J(E)$, one may study also the "self-Jung constant" $J_{s}(E)=\sup \left\{2 r_{A}(A) ; A \subset E\right.$ convex, diam $A=1\}$. Clearly $J_{s}(E) \geqq J(E)$. $E$ is said to have "normal structure" if for every such $A$ we have $r_{A}(A)<\operatorname{diam} A$. Thus $J_{s}(E)$ measures to what extent $E$ has "uniform normal structure". Bynum [3] introduced the "normal structure coefficient"; $N(E)=2 / J_{s}(E)$, and two other coefficients, $B S(E)$ and $W C S(E)$, analogously defined by the "asymptotic diameter" and the "asymptotic radius" of bounded, or 
weakly convergent, sequences in $E$, respectively, i.e.

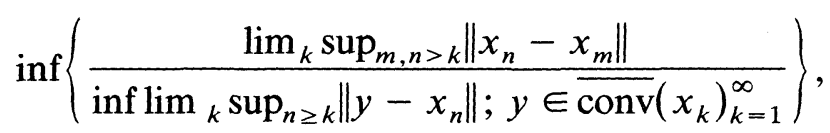

where the infinum is taken over all bounded nonconvergent sequences $\left(x_{n}\right) \subset E$ in the $B S(E)$ case, and over all weakly convergent, non-normconvergent sequences in the $W C S(E)$ case. Clearly $1 \leq N(E) \leq B S(E)$ $\leq W C S(E)$ and $W C S(E) \leq 2$ unless $E$ has the Schur property (i.e. unless in $E$ norm and weak sequential convergence coincide).

It is easy to see, and hinted in [3], that $B S(E)=\sup \{N(F) ; F \subset E$ separable $\}$ and $W C S(E)=\sup \{W C S(F) ; F \subset E$ separable $\}$.

In [15], Lim shows that $J_{s}(E)=\sup \left\{2 r_{A}(A) ; A \subset E\right.$ convex and separable, $\operatorname{diam} A=1$, hence $N(E)=B S(E)$ for every normed $E$. This can be further improved, using the following observations:

2.1. Proposition. (a) If $E$ is a dual Banach space, then

$$
J(E)=\sup \left\{2 r_{E}(K) ; K \subset E \text { finite, diam } K=1\right\} .
$$

(b) If $E$ is a reflexive Banach space, then

$$
J_{s}(E)=\sup \left\{2 r_{\text {conv } K}(K) ; K \subset E \text { finite, diam } K=1\right\} .
$$

Proof. (a) Let $A \subset E$ be any with $\operatorname{diam} A=1, r<r_{E}(A)$ any. Then $\bigcap_{x \in A} B(x, r)=\varnothing$ and by $\mathrm{w}^{*}$-compactness of the balls there is a finite $K=\left\{x_{1}, \ldots, x_{n}\right\} \subset A$ with $\bigcap_{x \in K} B(x, r)=\varnothing$, i.e. $r<r_{E}(K)$.

(b) Let $A \subset E$ be convex closed with $\operatorname{diam} A=1, r<r_{A}(A)$ any. Then $\cap_{x \in A} B(x, r) \cap A=\varnothing$ and by w-compactness of the balls and of $A$ there is a finite $K \subset A$ with $\bigcap_{x \in K} B(x, r) \cap \operatorname{conv} K \subset \bigcap_{x \in K} B(x, r) \cap A$ $=\varnothing$, i.e. $r<r_{\text {conv } K}(K)$.

2.2. Proposition. (Maluta, [16].) If $E$ is a non reflexive Banach space, then $J_{s}(E)=2$.

Proof. By a theorem of D. P. Milman and V. D. Milman [18] there is, in every nonreflexive Banach space $E$ and for every $\varepsilon>0$, a sequence $\left(x_{n}\right)_{n=1}^{\infty}$ in $E$ such that for every $m \geq 1$ and every $y^{\prime} \in \operatorname{conv}\left(x_{1}, \ldots, x_{m}\right)$, $y^{\prime \prime} \in \operatorname{conv}\left(x_{m+1}, x_{m+2}, \ldots\right)$ we have $1-\varepsilon<\left\|y^{\prime}-y^{\prime \prime}\right\|<1+\varepsilon$. Taking $A=\operatorname{conv}\left(x_{n}\right)_{n=1}^{\infty}$, one has $\operatorname{diam} A \leq 1+\varepsilon$ while $r_{A}(A) \geq 1-\varepsilon$.

2.3. Corollary. (a) (Lim) $J_{s}(E)=\sup \left\{2 r_{\operatorname{conv} A} A ; A \subset E\right.$ separable, $\operatorname{diam} A=1\}=\max \left\{J_{s}(F) ; F\right.$ a separable subspace of $\left.E\right\}$. 
(b) If $J_{s}(E)<2$, then $J_{s}(E)=\sup \left\{2 r_{\text {conv } K} K ; K \subset E\right.$ finite, diam $K=$ $1\}=\sup \left\{J_{s}(F) ; F\right.$ a finite dimensional subspace of $\left.E\right\}$.

(c) If $E$ has "uniform normal structure", so does every reflexive $G$ which is finitely representable in $E$ (i.e. such that for every finite dimensional subspace $F$ of $G$ and every $\varepsilon>0$ there is an isomorphism $T$ of $F$ onto a subspace of $E$ with $\|T\|\left\|T^{-1}\right\|<1+\varepsilon$ ).

Proof. Immediate from Propositions 2.1(b) and (2.2) and from the fact that every non reflexive Banach space contains a separable non reflexive subspace.

REMARK. It is not clear, however, from the above whether "uniform normal structure" is a superproperty, i.e. whether "reflexive" can be dropped in (c) or, equivalently, whether "uniform normal structure" implies superreflexivity.

We observe here that the (absolute) Jung constant $J(E)$ cannot be estimated from either side by the Jung constants of its subspaces in a similar way. Any space $E$ is a subspace of some $\mathscr{P}_{1}$-space $F=l_{\infty}(\Gamma)$ for some $\Gamma$ (e.g. the dual ball) and $J(F)=1$ while $J(E)$ can be any. Thus we may have $J(E)>J(F)$ when $E \subset F$. We cannot also get lower bounds for $J(E)$ by considering finite or separable subsets, as shown by:

2.4. Examples. (a) $J\left(c_{0}\right)=2$ by Proposition 1.1 (e.g. take $A=$ $\left\{(-1)^{n} e_{n} ; n=1,2, \ldots\right\}$, then $\left.\operatorname{diam} A=1=r_{c_{0}}(A)\right)$. However, for every finite $A=\left\{x_{1}, \ldots, x_{n}\right\} \subset c_{0}, \quad \bar{x}=\frac{1}{2}\left(\max _{1 \leq l \leq n} x_{i}-\min _{1 \leq i \leq n} x_{i}\right) \in c_{0}$ satisfies $r(\bar{x}, A)=\frac{1}{2} \operatorname{diam} A$.

(b) Let $\Gamma$ be an uncountable set, $E=\{x \notin m(\Gamma)$; spt $x$ countable $\}$ (where spt $x=\{\gamma ; x(\gamma) \neq 0\}$ ). $E$ is a closed subspace of $m(\Gamma)$, hence Banach. Every separable subset of $E$ is contained in a subspace of $m\left(\Gamma_{0}\right)$, where $\Gamma_{0} \subset \Gamma$ is countable (the union of the supports of a dense sequence). $m\left(\Gamma_{0}\right)$ is a subspace of $E$ isometric to $m=l_{\infty}$ which has Jung constant $J(M)=1$. On the other hand, let $\Gamma=\Gamma_{1} \cup \Gamma_{2}$, where $\Gamma_{1}, \Gamma_{2}$ are uncountable and disjoint, $A_{i}=\left\{x \in E ; 0 \leqq x \leqq x_{\Gamma_{1}}\right\}(i=1,2), A=A_{1} \cup\left(-A_{2}\right)$. It is easily seen that $\operatorname{diam} A=1$ but $r(A)=1$. Thus $J(E)=2$.

On the other hand, we have:

2.5. Proposition. Let $\left(E_{\alpha}\right)_{\alpha \in D}$ be a net of linear subspaces of the Banach space $E$, directed by inclusion, such that ${\overline{U_{\alpha \in D}}}_{E_{\alpha}}=E$. Then: (a) If $E$ is reflexive, then $J_{s}(E)=\sup _{\alpha} J_{s}\left(E_{\alpha}\right)=\lim _{\alpha \in D} J_{s}\left(E_{\alpha}\right)$.

(b) If $E$ is a dual space and each $E_{\alpha}$ admits a norm-1 linear projection $P_{\alpha}$, then $J(E)=\sup _{\alpha} J\left(E_{\alpha}\right)=\lim _{\alpha \in D} J\left(E_{\alpha}\right)$. 
Proof. If $P$ is a norm-1 projection of $E$ onto $F$, then for every $A \subset F$, $x \in E$ we have $r(P x, A) \leq r(x, A)$, hence $r_{F}(A)=r_{E}(A)$, thus $J(F) \leq$ $J(E)$. Therefore for every $\alpha \leq \beta$ we have $J_{s}\left(E_{\alpha}\right) \leq J_{s}\left(E_{\beta}\right) \leq J_{s}(E)$ or $J\left(E_{\alpha}\right) \leq J\left(E_{\beta}\right) \leq J(E)$, respectively. In either case it is enough to consider $A=\operatorname{conv}\left(x_{1}, \ldots, x_{n}\right) \in E$ with

$$
\frac{r_{A}(A)}{\operatorname{diam} A}>\frac{1}{2} J_{s}(E)-\varepsilon \text { or } \frac{r_{E}(A)}{\operatorname{diam} A}>\frac{1}{2} J(E)-\varepsilon,
$$

respectively. But taking $x_{1}^{\prime}, \ldots, x_{n}^{\prime} \in E_{\alpha}$ with $\left\|x_{t}-x_{\imath}^{\prime}\right\|<\varepsilon$ for $i=1, \ldots, n$ we get $A^{\prime}=\operatorname{conv}\left\{x_{1}^{\prime}, \ldots, x_{n}^{\prime}\right\} \subset E_{\alpha}$ (for some $\alpha$ ) satisfying, respectively,

$$
\frac{r_{A^{\prime}}\left(A^{\prime}\right)}{\operatorname{diam} A^{\prime}}>\frac{r_{A}(A)-\varepsilon}{\operatorname{diam} A+\varepsilon} \text { or } \quad \frac{r_{E}\left(A^{\prime}\right)}{\operatorname{diam} A^{\prime}}>\frac{r_{E}(A)-\varepsilon}{\operatorname{diam} A+\varepsilon}
$$

2.6. Corollary. For every $1 \leq p<\infty$ and every infinite dimensional $L_{p}(\mu)$ space we have

(a) $J_{s}\left(L_{p}(\mu)\right)=J_{s}\left(l_{p}\right)=\sup _{n} J_{s}\left(l_{p}^{n}\right)=\lim _{n} J_{s}\left(l_{p}^{n}\right)$ and

(b) $J\left(L_{p}(\mu)\right)=J\left(l_{p}\right)=\sup _{n} J\left(l_{p}^{n}\right)=\lim _{n} J\left(l_{p}^{n}\right)$.

Proof. For every measurable partition $D=\left\{D_{0}, D_{1}, \ldots, D_{n}\right\}$ of the measure space, with $0<\mu\left(D_{i}\right)<\infty$ for $i=1, \ldots, n$, the characteristic fucntions $\left\{\chi_{D_{1}}, \ldots, \chi_{D_{n}}\right\}$ span in $L_{p}(\mu)$ a subspace $F_{D}$ isometric to $l_{p}^{n}$, and admitting the norm- 1 projection $P_{D} f=\sum_{i=1}^{n}\left(\int_{D_{l}} f d \mu\right) \chi_{D_{l}} / \mu\left(D_{l}\right)$. The $F_{D}$ clearly form a net directed by inclusion whose union is dense in $L_{p}(\mu)$, so that we can apply Proposition 2.5.

In order to give lower bounds for $J$ and $J_{s}$ in $n$-dimensional spaces, consider " $(n, m, r)$-symmetric block designs", i.e. 0-1 symmetric $n \times n$ matrices $A=\left(a_{i j}\right)_{i, j=1}^{n}$ such that

$$
\sum_{j=1}^{n} a_{i j} a_{k J}= \begin{cases}m & \text { if } i=k \\ r & \text { if } i \neq k,\end{cases}
$$

where $n>m>0$ and $r$ is, necessarily, $m(m-1) /(n-1)$.

2.7. LEMMA. If $E$ is an n-dimensional space with a symmetric basis $\left(e_{k}\right)_{k=1}^{n}\left(i . e\right.$. such that $\left\|\sum_{k=1}^{n}\left|\alpha_{k}\right| e_{k}\right\|=\left\|\sum_{k=1}^{n} \alpha_{\pi(k)} e_{k}\right\|$ for all scalars $\alpha_{1}, \ldots, \alpha_{n}$ and all permutations $\pi$ of $\{1, \ldots, n\})$, and if there is an $(n, m, r)$-symmetric block design $\left(a_{i j}\right)_{i, j=1}^{n}$, then

$$
J(E) \geq 2 \min _{0 \leq \alpha \leq 1}\left\|(1-\alpha) \sum_{i=1}^{m} e_{i}+\alpha \sum_{i=m+1}^{n} e_{i}\right\|\left\|_{i=1}^{2(m-r)} e_{l}\right\|^{-1}
$$


and

$$
J_{s}(E) \geq 2\left\|\left(1-\frac{m}{n}\right) \sum_{i=1}^{m} e_{i}+\frac{m}{n} \sum_{i=m+1}^{n} e_{i}\right\|\left\|\sum_{i=1}^{2(m-r)} e_{i}\right\|^{-1} .
$$

If there is an ( $n, m, m / 2)$-symmetric block design (hence, necessarily, $m=(n+1) / 2)$, then also

$$
J_{s}(E) \geq\left\|\sum_{i=1}^{n} e_{i}\right\|\left\|\sum_{i=1}^{m} e_{i}\right\|^{-1} .
$$

Proof. Consider the points $x_{i}=\sum_{j=1}^{n} a_{i j} e_{j}$ and the sets $A=$ $\operatorname{conv}\left(x_{1}, \ldots, x_{n}\right)$ or $A_{0}=\operatorname{conv}\left(0, x_{1}, \ldots, x_{n}\right)$, respectively. By symmetry, center points are multiples of $\sum_{i=1}^{n} e_{i}$. Also,

$$
\min _{0 \leq \alpha \leq m / n} \max \left(\left\|(1-\alpha) \sum_{i=1}^{m} e_{l}+\alpha \sum_{i=m+1}^{n} e_{\imath}\right\|, \alpha\left\|\sum_{i=1}^{n} e_{i}\right\|\right)=\frac{1}{2}\left\|\sum_{i=1}^{n} e_{i}\right\| .
$$

2.8. CoROllaRy. If there is an $(n, m, r)$-symmetric block design then, for every $1 \leq p \leq \infty$, we have

$$
J\left(l_{p}^{n}\right) \geq\left(\frac{2^{p-1}(n-1)}{\|(m, n-m)\|_{q-1}}\right)^{1 / p} \quad\left(\text { where } \frac{1}{p}+\frac{1}{q}=1\right)
$$

(since the minimizing $\alpha$ for $p>1$ is $m^{1 / p-1} /\left(m^{1 / p-1}+(n-m)^{1 / p-1}\right)$ and for $p=1$ it is 1 if $m \geq 2 n$ and 0 if $m \leq 2 n)$, and

$$
J_{s}\left(l_{p}^{n}\right) \geq \frac{\left(2^{p-1}(n-1)\|(m, n-m)\|_{p-1}^{p-1}\right)^{1 / p}}{n} .
$$

If there is an ( $n, m, m / 2)$-symmetric block design, then

$$
J_{s}\left(l_{p}^{n}\right) \geq(2 n /(n+1))^{1 / p} .
$$

2.9. Lemma. There are $(n, m, r)$-symmetric block designs in each of the following cases:

(a) $n$ is any, $m=1, r=0$, or $m=n-1, r=n-2$.

(b) $n=2^{2 t}, m=2^{t-1}\left(2^{t}-1\right), r=2^{t-1}\left(2^{t-1}-1\right)$ or $m=2^{t-1}\left(2^{t}+1\right)$, $r=2^{t-1}\left(2^{t-1}+1\right)$.

(c) $n=2^{t}-1, m=2^{t-1}, r=2^{t-2}$, or $m=2^{t-1}-1, r=2^{t-2}-1$. 
Proof. For (a) take the unit matrix, $a_{i j}=\delta_{i j}$ or its complement $a_{i j}=1-\delta_{i j}$. For (b) define, inductively, $A_{0}=(1), B_{0}=(0)$,

$$
A_{t+1}=\left(\begin{array}{cccc}
B_{t} & A_{t} & A_{t} & A_{t} \\
A_{t} & B_{t} & A_{t} & A_{t} \\
A_{t} & A_{t} & B_{t} & A_{t} \\
A_{t} & A_{t} & A_{t} & B_{t}
\end{array}\right), \quad\left(B_{t+1}\right)_{i j}=1-\left(A_{t+1}\right)_{i j}
$$

For (c), let $W_{t}=\left(w_{i j}^{t}\right)_{i, j=1}^{2^{t}}$ be the Walsh matrix, defined inductively by $W_{0}=(1)$,

$$
W_{t+1}=\left(\begin{array}{rr}
W_{t} & W_{t} \\
W_{t} & -W_{t}
\end{array}\right),
$$

and consider $\left(\frac{1}{2}\left(1-w_{i j}^{t}\right)\right)_{i, j=2}^{2^{t}}$.

2.10. Corollary. (a) $J\left(l_{p}^{2^{t}}\right) \geq\left(\left(2^{t}-1\right) / 2^{t-1}\right)^{1 / p}$.

(b) $J_{s}\left(l_{p}^{n}\right) \geq 2^{p-1 / p}\left[(n-1)+(n-1)^{p}\right]^{1 / p} / n$.

(c) $J_{s}\left(l_{p}^{2^{t}-1}\right) \geq\left(\left(2^{t}-1\right) / 2^{t-1}\right)^{1 / p}$.

(d) $J\left(l_{p}\right) \geq 2^{1 / p}$.

(e) $J_{s}\left(l_{p}\right) \geq \max \left(2^{1 / p}, 2^{p-1 / p}\right)$.

((e) follows also from Corollary 2.6 and Bynum's estimate $W C S\left(L_{p}\right)$ $\leq \min \left(2^{p-1 / p}, 2^{1 / p}\right)$.

2.11. Corollary. (a) $J_{s}(E) \geq 2^{1 / p_{E}}$, where $p_{E}=\inf \left\{p ; l_{p}\right.$ is finitely represented in $E\}=$ the maximal "type" of $E$ in the sense of Maurey and Pisier [17]. Thus, if E has uniform normal structure, it is "B-convex" ([13]). In fact, stronger conditions are imposed on $E$ (cf. [1]).

(b) For every infinite-dimensional $E, J_{s}(E) \geq \sqrt{2}$ (Maluta, [16]) ( since $p_{E} \leq 2$ by Dvoretzky's theorem).

Now we observe some upper bounds.

2.12. Proposition. If $\operatorname{dim} E \leq n$, then $J_{s}(E) \leq 2 n /(n+1)$.

Proof. Given a convex $A \subset E$ with $\operatorname{diam} A=1$, take any $r<r_{A}(A)$. Then $\bigcap_{x \in A} B(x, r) \cap \bar{A}=\varnothing$ hence by Helly's theorem, there are $x_{0}, \ldots, x_{n} \in A$ with $\bigcap_{i=0}^{n} B\left(x_{i}, r\right) \cap A=\varnothing$. But, taking

$$
\bar{x}=\frac{1}{n+1} \sum_{i=0}^{n} x_{i} \in A,
$$


we have

$$
\begin{aligned}
\left\|\bar{x}-x_{j}\right\| & =\frac{1}{n+1}\left\|\sum_{i=0}^{n}\left(x_{i}-x_{j}\right)\right\|=\frac{1}{n+1}\left\|\sum_{i \neq j}\left(x_{i}-x_{j}\right)\right\| \\
& \leq \frac{1}{n+1} \max _{i \neq j}\left\|x_{i}-x_{j}\right\| \leq \frac{n}{n+1},
\end{aligned}
$$

hence $r<n /(n+1)$. Since $r<r_{A}(A)$ was arbitrary, $r_{A}(A) \leq n /(n+1)$. $\square$

$$
\begin{aligned}
& \text { If }\left(x_{0}, x_{1}, \ldots, x_{n}\right) \in E \text {, the " } n \text {-volume" of } \operatorname{conv}\left(x_{0}, \ldots, x_{n}\right) \text { is } \\
& V\left(x_{0}, \ldots, x_{n}\right)=\sup \left\{\operatorname{det}\left(\begin{array}{c}
1, f_{1}, \ldots, f_{n} \\
x_{0}, x_{1}, \ldots, x_{n}
\end{array}\right) ; f_{l} \in B\left(E^{*}\right), i=1, \ldots, n\right\} .
\end{aligned}
$$

Following Sullivan [21], we define the modulus of $n$-convexity of $E$,

$$
\begin{array}{r}
\delta_{E}^{(n)}(\varepsilon)=\inf \left\{1-\frac{1}{n+1}\left\|\sum_{i=0}^{n} x_{i}\right\| ; x_{i} \in B(E),\right. \\
\left.\quad i=0, \ldots, n, V\left(x_{0}, \ldots, x_{n}\right) \geq \varepsilon\right\}
\end{array}
$$

(so that

$$
\delta_{E}^{(1)}(\varepsilon)=\delta_{E}(\varepsilon)=\inf \left\{1-\left\|\frac{x_{0}+x_{1}}{2}\right\| ; x_{0}, x_{1} \in B(E),\left\|x_{0}-x_{1}\right\| \geq \varepsilon\right\}
$$

is the ordinary modulus of convexity). Sullivan showed that if $E$ is " $n$-uniformly convex", i.e. if $\delta_{E}^{(n)}(\varepsilon)>0$ for all $\varepsilon>0$, then $E$ is superreflexive and has normal structure. Bynum [3] observed that $J_{s}(E) \leq 2\left(1-\delta_{E}(1)\right)$. One can push this argument one step further:

\subsection{Proposition.}

$$
J_{s}(E) \leq 2 \min _{\varepsilon} \max \left(1-\delta_{E}^{(2)}(\varepsilon), \frac{2}{3} \varepsilon+\frac{1}{2}\right) .
$$

Proof. Let $A \subset E$ be convex with $\operatorname{diam} A=1$. Suppose $r_{A}(A)>r>$ $1-\delta_{E}^{(2)}(\varepsilon)$. Take $\eta>0$ and $x_{0}, x_{1} \in A$ with $\left\|x_{1}-x_{0}\right\|>1-\eta$ and $x_{2} \in A$ with

$$
\left\|x_{2}-\frac{x_{0}+x_{1}}{2}\right\|>r
$$

$x_{3} \in A$ with

$$
\left\|x_{3}-\frac{x_{0}+x_{1}+x_{2}}{3}\right\|>r .
$$


Translating, we may assume $x_{3}=0$. Take $f_{1} \in B\left(E^{*}\right)$ with

$$
f_{1}\left(x_{1}-x_{0}\right)>1-\eta \text { and } f_{2} \in B\left(E^{*}\right)
$$

with

$$
f_{2}\left(x_{2}-\frac{x_{0}+x_{1}}{2}\right)>r
$$

Then

$$
\begin{aligned}
V\left(x_{0}, x_{1}, x_{2}\right) & =\left|\begin{array}{ccc}
1 & 1 & 1 \\
f_{1}\left(x_{0}\right) & f_{1}\left(x_{1}\right) & f_{1}\left(x_{2}\right) \\
f_{2}\left(x_{0}\right) & f_{2}\left(x_{1}\right) & f_{2}\left(x_{2}\right)
\end{array}\right| \\
& =\left|\begin{array}{cc}
f_{1}\left(x_{1}-x_{0}\right) & f_{1}\left(x_{2}-\left(x_{0}+x_{1}\right) / 2\right) \\
f_{2}\left(x_{1}-x_{0}\right) & f_{2}\left(x_{2}-\left(x_{0}+x_{1}\right) / 2\right)
\end{array}\right| \\
& >(1-\eta) r-f_{1}\left(x_{2}-\frac{x_{0}+x_{1}}{2}\right) f_{2}\left(x_{1}-x_{0}\right) .
\end{aligned}
$$

But

$$
\begin{aligned}
f_{1}\left(x_{2}-\frac{x_{0}+x_{1}}{2}\right) & =f_{1}\left(x_{1}-x_{0}\right)+f_{1}\left(\frac{2 x_{2}+x_{0}}{2}-\frac{3 x_{1}}{2}\right) \\
& =f_{1}\left(x_{1}-x_{0}\right)+\frac{3}{2} f_{1}\left(\frac{2 x_{2}+x_{0}}{3}-x_{1}\right) \\
& >(1-\eta)-\frac{3}{2}=-\frac{1}{2}-\eta
\end{aligned}
$$

and also

$$
f_{1}\left(x_{2}-\frac{x_{0}+x_{1}}{2}\right)=-f_{1}\left(x_{1}-x_{0}\right)+f_{1}\left(\frac{2 x_{2}+x_{1}}{2}-\frac{3 x_{0}}{2}\right)<\frac{1}{2}+\eta .
$$

Similarly,

$$
f_{2}\left(x_{1}-x_{0}\right)=f_{2}\left(x_{2}-\frac{x_{0}+x_{1}}{2}\right)+f_{2}\left(\frac{3 x_{1}}{2}-\frac{2 x_{2}+x_{0}}{2}\right)>r-\frac{3}{2}
$$

and

$$
f_{2}\left(x_{1}-x_{0}\right)=-f_{2}\left(x_{2}-\frac{x_{0}+x_{1}}{2}\right)+f_{2}\left(\frac{x_{1}+2 x_{2}}{2}-\frac{3 x_{0}}{2}\right)<\frac{3}{2}-r .
$$

Thus

$$
\varepsilon \geq V\left(x_{0}, x_{1}, x_{2}\right)>(1-\eta) r-\left(\frac{1}{2}+\eta\right)\left(\frac{3}{2}-r\right) .
$$

Since $\eta>0$ was arbitrary, $3 r / 2-3 / 4 \leq \varepsilon$ or $r \leq 2 \varepsilon / 3+1 / 2$. 
If we use this estimate for $l_{2}$ we get $J_{s}\left(l_{2}\right) \leq 1.61$ (while $2\left(1-\delta_{l_{2}}(1)\right)=$ $\sqrt{3}$ and $\left.J_{s}\left(l_{2}\right)=\sqrt{2}\right)$. In any $E$, if $\delta_{E}^{(2)}(3 / 4)>0$, then $J_{s}(E)<2$.

2.14. Proposition. For every $n$ and every $\varepsilon>0$, we have $J_{s}(E) \leq$ $2 \max \left(1-(1-\varepsilon) / n ! \varepsilon, 1-\delta_{E}^{(n)}(\varepsilon)\right)$, so that if $\delta_{E}^{(n)}(1)>0$ then $E$ has uniform normal structure.

Proof. Let $A \subset E$ be convex with $\operatorname{diam} A=1$. Take any $r<r_{A}(A)$ and any $\eta>0$. Find $x_{0}, x_{1} \in A$ with $\left\|x_{0}-x_{1}\right\|>1-\eta$ and $x_{k} \in A$, $k=2,3, \ldots, n+1$ with $\left\|x_{k}-k^{-1} \sum_{i=0}^{k-1} x_{i}\right\|>r$ (such $x_{k}$ exist since $k^{-1} \sum_{i=0}^{k-1} x_{i} \in A$ and $\left.r_{A}(A)>r\right)$. Translate to get $x_{n+1}=0$, so that $x_{i} \in$ $B(E), i=0, \ldots, n$. Find $f_{1} \in B\left(E^{*}\right)$ with $f_{1}\left(x_{1}-x_{0}\right)>1-\eta$ and $f_{k} \in$ $B\left(E^{*}\right), k=2, \ldots, n$, with $f_{k}\left(x_{k}-k^{-1} \sum_{i=0}^{k-1} x_{i}\right)>r$. Consider

$$
\begin{aligned}
& V\left(x_{0}, \ldots, x_{n}\right) \geq \operatorname{det}\left(\frac{1, f_{1}, \ldots, f_{n}}{x_{0}, x_{1}, \ldots, x_{n}}\right) \\
& \quad=\operatorname{det}\left(\begin{array}{c}
f_{1}, f_{2}, f_{3}, \ldots, f_{n} \\
x_{1}-x_{0}, x_{2}-\frac{1}{2}\left(x_{0}+x_{1}\right), x_{3}-\frac{1}{3} \sum_{i=0}^{2} x_{i}, \ldots, x_{n}-\frac{1}{n} \sum_{i=0}^{n-1} x_{i}
\end{array}\right) .
\end{aligned}
$$

All the entries in the last determinant have absolute value $\leq 1$, but the subdiagonal ones, $f_{m}\left(x_{k}-k^{-1} \sum_{i=0}^{k-1} x_{i}\right)$ for $m>k$, are small for $r$ close to 1: since $m^{-1} \sum_{i=0}^{m-1} f_{m}\left(x_{m}-x_{i}\right)>r$ and $\left|f_{m}\left(x_{m}-x_{i}\right)\right| \leq 1$, we have 1 $m(1-r)<f_{m}\left(x_{m}-x_{i}\right) \leq 1$ for $i<m$, hence

$$
\left|f_{m}\left(x_{k}-x_{l}\right)\right|=\left|f_{m}\left(x_{m}-x_{i}\right)-f_{m}\left(x_{m}-x_{k}\right)\right|<m(1-r)
$$

and

$$
\left|f_{m}\left(x_{k}-\frac{1}{k} \sum_{i=0}^{k-1} x_{i}\right)\right|=\left|\frac{1}{k} \sum_{i=0}^{k-1} f_{m}\left(x_{k}-x_{i}\right)\right|<m(1-r),
$$

too. Thus

$$
V\left(x_{0}, \ldots, x_{n}\right)>(1-\eta) r^{n-1}-(n !-1) n(1-r)>\varepsilon
$$

provided $r>1-(1-\varepsilon-\eta) / n ! n$. Therefore for such $r$ we must have

$$
r<\left\|1 /(n+1) \sum_{i=0}^{n} x_{i}\right\|<1-\delta_{E}^{(n)}(\varepsilon) .
$$

Since $\eta>0$ and $r<r_{A}(A)$ were arbitrary, we get

$$
r_{A}(A) \leq \max \left(1-\delta_{E}^{(n)}(\varepsilon), 1-(1-\varepsilon) / n ! n\right) .
$$


REMARK. The rough estimate we used above can be improved, but since the computation of $\delta_{E}^{(n)}$ seems to be quite complicated, it is not clear whether finer estimates will yield more results.

Lim [15] gave the following upper bound for $J_{s}\left(l_{p}\right), p>2$ :

$$
J_{s}\left(l_{p}\right) \leq 2\left(1+\frac{1+t^{p-1}}{(1+t)^{p-1}}\right)^{-1 / p},
$$

where $0 \leq t \leq 1$ solves $(p-2) t^{p-1}+(p-1) t^{p-2}=1$.

Maluta [16] defined another related constant for a normed $E$ :

$D(E)=\sup \left\{\lim _{k} \sup _{n \geq k} d\left(x_{n+1}, \operatorname{conv}\left(x_{i}\right)_{i=1}^{n}\right) ;\left(x_{n}\right) \subset E, \operatorname{diam}\left(x_{n}\right)_{n=1}^{\infty}=1\right\}$, and showed that:

(i) $D(E)=\sup \{D(F) ; F \subset E$ separable $\}$;

(ii) $D(E)=0$ if and only if $E$ is finite-dimensional.

(iii) If $D(E)<1$ then the Banach space $E$ is reflexive and has normal structure (but $E=\left(\sum \oplus l_{n}\right)_{2}$ is reflexive and has normal structure although $D(E)=1)$.

(iv) $2 D(E) \leq J_{s}(E)$ and, if $E$ is reflexive, $D(E) \leq 1 / W C S(E)$.

Maluta asked if $D(E)=1 / W C S(E)$ for every reflexive $E$. She showed that this is the case for $l_{p}$, i.e. $D\left(l_{p}\right)=2^{-1 / p}$ (Bynum showed $\operatorname{WCS}\left(l_{p}\right)=$ $\left.2^{1 / p}\right) ; D\left(\left(\sum \oplus l_{\infty}^{n}\right)_{2}\right)=2^{-1 / 2}$ (Bynum showed $W C S\left(\left(\sum \oplus l_{\infty}^{n}\right)_{2}\right)=\sqrt{2}>$ $\left.1=N\left(\left(\sum \oplus l_{\infty}^{n}\right)_{2}\right)\right)$. For the space $l_{p, 1}$, i.e. $l_{p}$ with the norm $\|x\|_{p, 1}=$ $\left\|x^{+}\right\|_{p}+\left\|x^{-}\right\|_{p}$, which is of special interest since in it $\delta(1)=0$, one still has $D\left(l_{p, 1}\right)=1 / W C S\left(l_{p, 1}\right)=2^{-1 / p}$. We can give an affirmative answer to Maluta's question in the case that $E$ satisfies the (weak) Opial condition: $w_{n} \stackrel{\mathrm{w}}{\rightarrow} 0 \Rightarrow \liminf \left\|x_{n}-x\right\| \geq \liminf \left\|x_{n}\right\| \forall x \neq 0$ [19]. The $l_{p}$ spaces $(1<$ $p<\infty)$ satisfy this condition, but the $L_{p}[0,1]$ spaces do not, unless $p=2$.

2.15. Proposition. If $E$ satisfies Opial's condition, then $D(E) \geq$ $1 / W C S(E)$.

Proof. For any $0 \leq r<1 / W C S(E)$, we can find $\left(x_{n}\right) \subset E$ with $x_{n} \stackrel{\mathrm{w}}{\rightarrow} 0, \operatorname{diam}\left(x_{n}\right)=1$ and $\lim \sup \left\|x_{n}-x\right\|>r$ for every $x \in \overline{\operatorname{conv}}\left(x_{n}\right)$. In particular, $\lim \sup \left\|x_{n}\right\|>r+\varepsilon$ for some $\varepsilon>0$, so that we can take a subsequence $\left(x_{n}^{\prime}\right)$ with $\left\|x_{n}^{\prime}\right\|>r+\varepsilon, \forall n$. By Opial's condition we have $\liminf \left\|x_{n}^{\prime}-x\right\| \geq r+\varepsilon, \forall x$. Let $n_{1}=1$. If $n_{1}, \ldots, n_{k}$ have been chosen, take a finite $\varepsilon / 2$-net, $\left(y_{1}, \ldots, y_{m_{k}}\right)$, for $\operatorname{conv}\left(x_{n}^{\prime}, \ldots, x_{n_{k}}^{\prime}\right)$, and find $n_{k+1}$ so that $\left\|x_{n}^{\prime}-y_{j}\right\|>r+\varepsilon / 2$ for every $n \geq n_{k+1}, j \leq m_{k}$. Then $d\left(x_{n_{k+1}}^{\prime}, \operatorname{conv}\left(x_{n_{l}}^{\prime}\right)_{i=1}^{k}\right)>r$, so that $D(E) \geq r$. 
The parameters $J(E), 2 D(E)$ and $R(E)([1])$, although all of them between 1 and $J_{s}(E)$, are incomparable even for reflexive infinite dimensional spaces:

\subsection{EXAMPLES.}

(a) $J\left(l_{2}\right)=2 D\left(l_{2}\right)=\sqrt{2}>1=R\left(l_{2}\right)$.

(b) $E=\left(\sum \oplus l_{\infty}^{n}\right)_{2}$. Here $J(E)=1 ; 2 D(E)=\sqrt{2}$ and $R(E)=2$.

(c) $E=\left(\sum \oplus l_{1}^{n}\right)_{2}$. Here $2 D(E)=\sqrt{2}$ again, but $J(E)=R(E)=2$.

In concluding, we remark that none of the convexity properties $J(E)<2, J_{s}(E)<2, W C S(E)>1$ or $D(E)<1$ is isomorphy invariant. In fact, the "best" spaces have "worst" equivalent renormings. For $J$ this follows from Proposition $1.1(m=C(\beta N)$ has a maximal subspace 2-isomorphic to it of the type $C(T), T$ non-Stonian). For $J_{s}, W C S$ or $D$, it was observed by Maluta that $D\left(l_{2},\|\|_{J}\right)=1$, where $\|x\|_{J}=$ $\max \left(\|x\|_{2}, \sqrt{2}\|x\|_{\infty}\right)$.

\section{REFERENCES}

[1] D. Amir and C. Franchetti, The radius ratio and convexity properties in normed linear spaces, Trans. Amer. Math. Soc., 282 (1984), 275-291.

[2] F. Bohnenblust, Convex regions and projections in Minkowski spaces, Ann. Math., 39 (1938), 301-308.

[3] W. L. Bynum, Normal structure coefficients for Banach spaces, Pacific J. Math., 86 (1980), 427-436.

[4] L. Danzer, B. Grünbaum and V. Klee, Helly's theorem and its relatives, Convexity, Proc. Symp. Pure Math., 7 (1963), Amer. Math. Soc., 101-180.

[5] W. Davis, A characterization of $\mathscr{P}_{1}$-spaces, J. Approx. Theory, 21 (1977), 315-318.

[6] C. Franchetti, Restricted centers and best approximation in $C(Q)$, Ann. Fac. Sci. de Kinshasa, Zaire, 3 (1977), 35-45.

[7] Relations between the Jung constant and a certain projection constant in Banach spaces, Ann. Univ. Ferrara, 23 (1977), 39-44.

[8] _ Projections onto hyperplanes in Banach spaces, J. Approx. Theory, 38 (1983), 319-323.

[9] D. Giesy, On a convexity condition in normed linear spaces, Trans. Amer. Math. Soc., 125 (1966), 114-146.

[10] B. Grünbaum, On some covering and intersection properties in Minkowski spaces, Pacific J. Math., 9 (1959), 487-494.

[11] R. Holmes, A course on Optimization and Best Approximation, Lecture Notes in Mathematics, Springer Verlag, Berlin 1972.

[12] H. W. E. Jung, Über die kleinste Kugel, die eine räumliche Figur einschlisst, J. Reine Angew. Math., 123 (1901), 241-257.

[13] V. Klee, Circumspheres and inner products, Math. Scand., 8 (1960), 363-370.

[14] K. Leichtweiss, Zwei Extremalprobleme der Minkowski Geometrie, Math. Zeitschrift, 62 (1955), 37-49.

[15] T. C. Lim, On normal structure coefficient and bounded sequence coefficient, to appear in Proc. Amer. Math. Soc. 
[16] E. Maluta, Uniformly normal structure and related coefficients, Pacific J. Math., 111 (1984), 357-369.

[17] B. Maurey and G. Pisier, Séries de variable aléatoires vectorielles independantes et propriétés geométriques des espaces de Banach, Studia Math. 58 (1976), 45-90.

[18] D. P. Milman and V. D. Milman, The geometry of nested families with empty intersection - structure of the unit sphere of a nonreflexive space, Amer. Math. Soc. Transl., (2) 85 (1969), 233-243.

[19] Z. Opial, Weak convergence of the sequence of successive approximations for nonexpansive mappings, Bull. Amer. Math. Soc., 73 (1967), 591-597.

[20] N. Routledge, A result in Hilbert space, Quart. J. Math., 3 (1952), 12-18.

[21] F. Sullivan, $A$ generalization of uniformly rotund Banach spaces, Canad. J. Math., 31 (1979), 628-636.

Received February 14, 1983 and in revised form December 6, 1983.

TEL-AVIV UNIVERSITY

TEL-AVIV, ISRAEL 



\title{
PACIFIC JOURNAL OF MATHEMATICS EDITORS
}

DONALD BABBITT (Managing Editor)
University of California
Los Angeles, CA 90024
CHARLEs R. DEPrIMA
California Institute of Technology
Pasadena, CA 91125
R. FINN
Stanford University
Stanford, CA 94305

DoNAld BABBitT (Managing Editor)

Hermann FlaschKa

University of Arizona

Tucson, AZ 85721

RAmesh A. Gangolli

University of Washington

Seattle, WA 98195

ROBION KIRBY

University of California

Berkeley, CA 94720

C. C. MOORE

University of California

Berkeley, CA 94720

\author{
Hugo Rossi \\ University of Utah \\ Salt Lake City, UT 84112 \\ H. SAMELSON \\ Stanford University \\ Stanford, CA 94305 \\ HAROLD STARK \\ University of California, San Diego \\ La Jolla, CA 92093
}

ASSOCIATE EDITORS

R. ARENS

E. F. BECKENBACH
(1906-1982)

B. H. NEUMANN

F. WOLF

K. Yoshida

\section{SUPPORTING INSTITUTIONS}

UNIVERSITY OF ARIZONA

UNIVERSITY OF BRITISH COLUMBIA

CALIFORNIA INSTITUTE OF TECHNOLOGY

UNIVERSITY OF CALIFORNIA

MONTANA STATE UNIVERSITY

UNIVERSITY OF NEVADA, RENO

NEW MEXICO STATE UNIVERSITY

OREGON STATE UNIVERSITY
UNIVERSITY OF OREGON

UNIVERSITY OF SOUTHERN CALIFORNIA

STANFORD UNIVERSITY

UNIVERSITY OF HAWAII

UNIVERSITY OF TOKYO

UNIVERSITY OF UTAH

WASHINGTON STATE UNIVERSITY

UNIVERSITY OF WASHINGTON 


\section{Pacific Journal of Mathematics}

\section{Vol. 118, No. $1 \quad$ March, 1985}

Dan Amir, On Jung's constant and related constants in normed linear spaces ...1 Abdul Aziz, On the location of the zeros of certain composite polynomials . . 17 Joseph Barback, On hereditarily odd-even isols and a comparability of

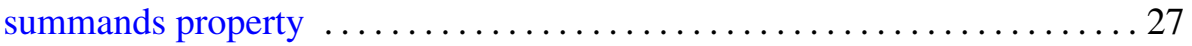

Matthew G. Brin, Klaus Johannson and Peter Scott, Totally peripheral 3-manifolds ........................................ 37

Robert F. Brown, A topological bound on the number of distinct zeros of an

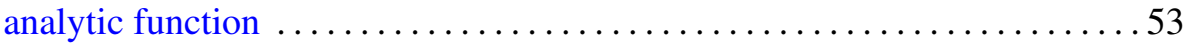

K. C. Chattopadhyay, Not every Lodato proximity is covered .......... 59

Beverly Diamond, Some properties of almost rimcompact spaces . .......63 63

Manfred Dugas and Rüdiger Göbel, On radicals and products . ......... 79

Abdelouahab El Kohen, A hyperbolic problem .................. 105

Harry Gonshor, Remarks on the Dedekind completion of a nonstandard model of the reals ................................... 117

William H. Kazez, On equivalences of branched coverings and their action

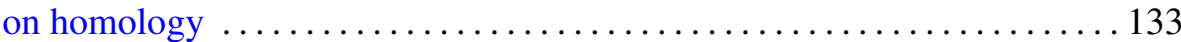

Darrell Conley Kent, On the Wallman order compactification .......... 159

Martin Andrew Magid, Lorentzian isoparametric hypersurfaces . . . . . . 165

Milan Miklavčič, Stability for semilinear parabolic equations with noninvertible linear operator

Richard Dean Neidinger and Haskell Paul Rosenthal, Norm-attainment of linear functionals on subspaces and characterizations of Tauberian operators

Johannes Vermeer, Closed subspaces of $H$-closed spaces 